\title{
Changes in Aggregate Form, Size and Flexibility Along Phase Sequences in Lyotropic Liquid Crystals
}

\author{
Lia Queiroz do Amaral \\ Instituto de Física da Universidade de São Paulo, \\ C.P. 66318, São Paulo, SP, 05315-970, Brazil
}

Received on 14 November, 2001

\begin{abstract}
A comparison is made between existing theories for self-assembling systems and experimental phase diagrams of complex systems made up of amphiphile/water/additives, with emphasis on phase transitions by changes in concentration. Evidence for different types of hexagonal $(\mathrm{H})$ phases, in systems with detergent and lipid amphiphiles, are reviewed. It is shown that characteristics of the $\mathrm{H}$ phase and of the phase sequence (cubic phases with lipids and nematic phases with detergents) are revealed through the exponent defining the variation of the hexagonal parameter with amphiphile concentration. Emphasis is also given to results obtained in the ternary system sodium dodecyl (lauryl) sulfate / water / decanol, which exhibits the phase sequence isotropic (I) - H - nematic cylindrical $\left(\mathrm{N}_{c}\right)$ - [nematic biaxial $\left.\left(\mathrm{N}_{b}\right)\right]$ - nematic discotic $\left(\mathrm{N}_{d}\right)$ - lamellar $(\mathrm{L})$, with co-surfactant addition. Existing theories for self-assembling systems of rigid and flexible rods predict the phase sequence I - $\left(\mathrm{N}_{c}\right)-\mathrm{H}$ as a function of increased particle volume fraction $v_{p}$, with a triple point separating $\mathrm{I}-\mathrm{H}$ and $\mathrm{I}-\mathrm{N}_{c}-\mathrm{H}$ phase sequences. Possible reasons for the non-trivial experimental $\mathrm{I}-\mathrm{H}-\mathrm{N}_{c}$ inversion in phase sequence are discussed. A complex path through the phase diagram is able to explain the experimental results in terms of changes in micellar growth and flexibility induced by the co-surfactant. The role of the surfactant parameter, that expresses the curvature of the polar-apolar interfaces, explains much of the observed behavior, including the $\mathrm{N}_{c}-\mathrm{N}_{d}$ transition with increase in decanol / amphiphile molar ratio.
\end{abstract}

\section{Introduction}

The complex polymorfism of systems made of amphiphile / water / additives (salt and / or alcohol) has been extensively investigated over the last decades $[1,2]$. Such polymorfism exists because the amphiphile molecules in solution self-assembly into aggregates of different forms and symmetries, due to the tendency of spontaneous formation of interfaces between polar and apolar groups. The formation of the aggregates is controlled by the surfactant parameter [3] $p_{o}=v / a l$, with $v, a$ and $l$, being, respectively, the volume of the chain, the polar head area at the interface (which depends also on the amount of bound water) and the effective length of the chain. Molecules with $p_{o}=1 / 3,1 / 2$ and 1 have tendency to form, respectively, spherical, cylindrical and planar micelles. In amphiphiles with a single hydrocarbon chain (as detergents, with $p_{o} \sim 1 / 3$ ) the interfaces may be closed and highly curved, with formation of micelles (direct in the water rich side of the phase diagram, inverted in the water poor side) and separation of hydrocarbon and water moieties. Micelles of initially spheroidal shape (when formed at the critical micellar concentration) may grow to cylindrical forms with increase in amphiphile concentration, and conse- quent decrease in $p_{o}$ due to decrease of bound water. Lamellar phases with planar interfaces or complex bicontinuous phases, with minimal curvature interfaces, may also occur in the intermediate zone of the phase diagram. Amphiphiles with two hydrocarbon chains (as phospholipids, with $p_{o} \sim 1$ ), instead, have tendency of forming vesicles (closed membranes with water inside and outside) due to their difficulty in forming highly curved interfaces. Such supra-molecular aggregates may have or not long distance order, giving rise to isotropic and liquid-crystalline phases, before reaching crystalline phases. Changes in the micellar shape and in the separating interfaces accompany or trigger the phase transitions as a function of concentration and temperature.

We shall here concentrate on amphiphiles with a single carbon chain, and on phase transitions induced by changes in concentration. The typical phase sequence with increasing amphiphile concentration is I (isotropic) - H (hexagonal) - L (lamellar). Intermediate phases between $\mathrm{H}$ and $\mathrm{L}$, with long-range positional order, may occur in binary systems. Alternatively, complex cubic phases (Q) [4] may also appear, that may be constituted either of micellar globules (in 
I-Q-H sequences) or of bicontinuous arrays (in H-Q-L sequences). Ternary systems, with addition of a long chain alcohol, may lead instead to nematic (N) domains between $\mathrm{H}$ and $\mathrm{L}$ phases (or directly from I phase), with uniaxial [5-7] (cylindrical $\mathrm{N}_{c}$ and discotic $\mathrm{N}_{d}$ ) and biaxial [8] $\left(\mathrm{N}_{b}\right)$ phases having only long-range orientational order. The role of alcohol in changing the aggregation process, leading to islands of nematic phases in ternary phase diagrams, is here discussed. The characteristics of $\mathrm{H}$ phases seem also to depend on the particular phase sequence where it appears. In this paper we shall review the work we have made on such phases over the last decade, with several collaborators.

Since the discovery of $\mathrm{H}$ phases, their structure has been proposed [1] to consist of "infinite" cylindrical micelles with a two-dimensional positional order in the plane perpendicular to the cylinder axes, based on the fact that only (hk0) diffractions were observed, without defined values for the $\mathrm{c}$ parameter, together with the indication of very long micelles by other techniques, as NMR. During the last decade, however, we have gathered evidence showing that in fact different structures exist, which seem to correlate with the specific phase sequence. Information on such structures is given by the exponent of the variation of the hexagonal parameter with concentration. Results obtained [9-13] with detergent and lipid amphiphiles are here reviewed.

We focus also in this paper on the analysis of results obtained [14-17] in the system sodium dodecyl (lauryl) sulfate (SLS) / water / decanol (which is a good example [18-20] of detergent behavior). The phase sequence I - H - $\mathrm{N}_{c}-\left(\mathrm{N}_{b}\right)-\mathrm{N}_{d}-\mathrm{L}$, obtained with increase in the decanol / amphiphile molar ratio $\left(M_{d}\right)$, is analyzed in particular. The results are confronted with statistical mechanical theories [21-23] of self-assembly that predict instead the phase sequences I - $\mathrm{N}_{c}-\mathrm{H}$ or I - $\mathrm{N}_{d}-\mathrm{L}$, depending on the symmetry of the aggregates (cylinders or discs, respectively). We consider also a recent result [24] obtained through an approach based on bending energy considerations, which is able to account for shape transformations at the $\mathrm{N}_{c}-\mathrm{N}_{d}$ transition, with increase of decanol content in systems with mixed micelles.

\section{Occupancy of the hexagonal cell}

As analyzed in our previous papers [9,11-13], for a spherocylinder (SC) of radius $R$ and total length $L$ in an hexagonal cell with parameters $a$ and $c$, the usual condition of equal density at the microscopic and macroscopic levels implies that the static structure (averaged in time) satisfies the relation

$$
(L / c)_{S C}=(2 \sqrt{ } 3 / \pi) c_{v}(a / 2 R)^{2}(\mu) /(\mu-1 / 3),
$$

where $\mu=L / 2 R$ is the particle anisometry (equal to one for spheres) and $c_{v}$ is the volume fraction of the total amphiphile moiety. Note that in the case of micelles the existence of bound water may make $c_{v}$ different than the particle volume fraction $v_{p}$. For long $\mathrm{SC}$ the cylinder (or rod) limit is obtained, and the factor $\mu /(\mu-1 / 3)$, that depends on the anisometry, becomes 1. This limit can be used in order to obtain $L / c$ values in the $\mathrm{H}$ phase, if micelles have large but unknown $\mu$ values [13].

Furthermore, in the case of micelles, as studied here, care must be taken in the definition of the $R$ and $L$ values in Eq. (1). They may refer to "effective" radius $R_{e f f}$ and length $L_{e f f}$, of the "particle without water", related to $c_{v}$. To substitute $v_{p}$ for $c_{v}$ in Eq. (1) it would be necessary to use $R_{t o t}$ and $L_{\text {tot }}$ of the "particle", including bound water. Alternatively, one can consider the paraffinic micelle and the values in Eq. (1) become $R_{\text {par }}, L_{\text {par }}$ and $c_{v p a r}$, where $c_{v p a r}$ is referred to as the paraffinic volume fraction. Moreover the following relationship is valid [13]:

$$
\left(R_{\mathrm{eff}} / R_{\mathrm{par}}\right)^{3}=\left(c_{v} / c_{\mathrm{vpar}}\right)\left(\mu_{\mathrm{par}}-1 / 3\right) /\left(\mu_{\mathrm{eff}}-1 / 3\right)
$$

For long micelles the differences between $L_{\text {eff }}, L_{\text {par }}$, $L_{\text {tot }}$ may be neglected, but not the differences between the respective $R$ values.

In these $\mathrm{H}$ phases no unique $c$ value exists, because of polydispersity of micellar length, a necessary condition for the occurrence of direct I-H phase transition [21], but "average values" are meaningful. As we shall see, the behavior of the $\mathrm{H}$ phase and of phase sequences depends on the relations between $a$ and $c$. In case of finite micelles and uniform decrease in inter-particle distances in all three dimensions, the anisometry of the particle exists also in the cell and one expects:

$$
L / c=2 R / a
$$

In case of "infinite" micelles one expects instead:

$$
L / c=1
$$

The structure of the $\mathrm{H}$ phase, and particularly its evolution with increased concentration, will depend on which Eq. (3) is valid for the particular system, and on the fact that $L$ may be a constant within the range of the $\mathrm{H}$ phase or may vary with concentration. When Eq. (3a) applies and the $L$ value is constant, it results $a \sim c_{v}^{-1 / 3}$. When Eq. (3b) applies, it results $a \sim c_{v}^{-1 / 2}$. In the general case, if it is experimentally observed $a=A c_{v}^{-x}$, the condition given by Eq. (3a) leads [11] to an equation that expresses the variation of the anisometry with concentration:

$$
\mu=\left[3-3 K c_{v}^{-(1-3 x)}\right]^{-1},
$$

where $K=(2 \sqrt{ } 3 / \pi)(A / 2 R)^{3}$. 
For cylinders with constant radius, $K$ is a constant. For $x=1 / 3$, the anisometry is also a constant, given by Eq. (4). But if $x<1 / 3, \mathrm{Eq}$. (4) gives the form of micellar growth with concentration, under the condition of Eq. (3a). The function $K c_{v}^{-(1-3 x)}$ varies from $2 / 3$ to 1 as $\mu$ varies from 1 (sphere) to $\infty$. However, as the limit of infinite cylinder approaches, Eq. (3a), and also Eq. (4), are no longer valid, and the condition given by Eq. (3b) is approached.

The validity of Eq. (3) for a particular system can be analyzed [11] through comparison of values $L / c$, obtained from Eq. (1) under the hypothesis of long rods (anisometry factor $=1$ ), and $2 R / a$, using an estimated $R$ value and the known $a$ and $c_{v}$ values. If $L / c<2 R / a$, there is indication of short rods, when the anisometry factor of Eq. (1) (always $<1$ ) cannot be neglected. But $L / c>2 R / a$ indicates a breakdown of Eq. (3a).

It has been also theoretically shown, in statistical mechanics treatment of self-assembly systems [21,22], that the exponents $1 / 3$ and $1 / 2$ hold, respectively, for finite rigid rods and for long flexible rods (with end-toend contact or with persistent length on the order of c). We shall therefore refer to conditions $L / c=2 R / a$ and $L / c=1$, Eq. (3), as characteristic of "finite/rigid" and "infinite/flexible" rods, respectively. In the case of flexible objects Eq. (1) does not hold in strict sense, but it is always possible to obtain an "apparent $L / c$ value" which satisfies Eq. (1) for rods.

\section{The $\mathbf{H}$ phase in different sys- tems}

In the binary SLS/water system the interval of the explored $c_{v}$ values, within the $\mathrm{H}$ domain, was sufficiently large and allowed us to unambiguously define [9] a $1 / 3$ behavior, typical of finite rigid rods with constant anisometry. A previous study [25] of the I-H phase transition had shown that the cylinder growth does not occur within the I phase, but at the transition itself, a view consistent with the statistical theories published later [21]. A detailed structural study [13] with careful analysis of the electron density maps obtained from diffraction intensities confirmed finite, although long, rods in the $\mathrm{H}$ phase of the binary SLS/water system.

Further studies in other amphiphile systems evidenced that the exponent depends on the particular phase sequence, being $1 / 2$ in a system with a neighboring cholesteric phase [10] and smaller than $1 / 3$ when a neighboring cubic phase is present in lipids [11,12]. For OLPC (oleoyl-lyso-phosphatidil-choline) / water system, with $\mathrm{I}-\mathrm{H}-\mathrm{Q}_{b}$ (cubic bicontinuous) - L phase sequence, the growth of the lipid micelles, obtained from Eq. (4), was clearly followed along the $\mathrm{H}$ domain [11], with anisometry near 1 at the $\mathrm{I}-\mathrm{H}$ transition up to $\infty$ at the $\mathrm{H}-\mathrm{Q}_{b}$ transition. The structures of $\mathrm{H}$ and $\mathrm{Q}$ phases of three lipids, OLPC, PaLPC (palmitoyl-lyso- phosphatidyl-choline), with I- $\mathrm{Q}_{m}$ (cubic micellar) - H - L phase sequence, and DTAC (dodecyl-trimethyl ammonium chloride), with $\mathrm{I}-\mathrm{Q}_{m}-\mathrm{H}-\mathrm{Q}_{b}-\mathrm{L}$ phase sequence have been detailed analyzed [12]. Micellar growth was verified along the $H$ phases of these three lipids (exponent $\mathrm{x}<1 / 3$ ) and epitaxial relationships at both $\mathrm{Q}_{m}-\mathrm{H}$ and $\mathrm{H}-\mathrm{Q}_{b}$ phase transitions could be demonstrated [12]. Micelles are small after I - H transition, larger after $\mathrm{Q}_{m}-\mathrm{H}$ transition, "infinite" before $\mathrm{H}-\mathrm{L}$ transition and have even $L / c>1$ before $\mathrm{H}-\mathrm{Q}_{b}$ transition.

A study of the $\mathrm{I}-\mathrm{Q}_{m}$ transition, that precedes the $\mathrm{Q}_{m}-\mathrm{H}$ transition in PaLPC revealed [26,27] that micelles remain spheroidal and do not grow to $\mathrm{SC}$ in the I phase, due to the larger surfactant parameter, and also how the micellar short-range order of the I phase transforms into the micellar cubic order due to close packing of the spheroidal micelles. For SLS micelles, a transformation of spheroidal into $\mathrm{SC}$ polydisperse micelles occurs [28] still in the I phase, due to the smaller surfactant parameter, preparing the $\mathrm{I}-\mathrm{H}$ phase transition. The role of the surfactant parameter in promoting aggregate transformations will be further discussed later on.

The picture that emerges from these studies follows a pattern. In the charged detergent SLS, micelles are already small SC in the I phase, and grow at the I$\mathrm{H}$ transition, due to the "orientation induced growth" mechanism [21], but have afterwards a constant size, until distortion of the micellar unit induces phase transitions leading finally to the $\mathrm{L}$ phase. In neutral lipids, growth does not occur at the transition to the $\mathrm{H}$ phase, but more slowly in the whole $\mathrm{H}$ range. In cases where an "excess growth" occurs, revealed by the condition $L / c>1$ when Eq. (1) is used (probably related to undulations of the micelle surface), the transition is towards a bicontinuous cubic phase $[11,12]$, while a H-L transition is observed when the limit $L / c=1$ is not over-passed. It is not yet possible to conclude whether these differences between charged detergents and neutral lipids are due directly to the soft inter-micellar Coulomb interaction or to differences in water binding.

The existence of a nematic (or cholesteric) domain seems to correlate with a neighbor $\mathrm{H}$ phase with exponent $\mathrm{x}=1 / 2$ (infinite/flexible objects), what is intriguing, since the lyotropic nematic phases are expected to correspond to small micelles [7], and a minimum rigidity is also necessary for the $\mathrm{N}$ phases [23]. Thus, the role of decanol in promoting the appearance of $\mathrm{N}$ phases deserves particular attention.

\section{The H phase neighbour to $N_{c}$ phase}

Addition of decanol to several detergent / water systems leads to a nematic domain. We shall here con- 
centrate in the $\mathrm{N}$ domain of the ternary system SLS / water / decanol [18-20]. The phase sequence depends on concentration and temperature and may be $\mathrm{I}-\mathrm{N}_{c}$ or I - H - $\mathrm{N}_{c}$ [14-16]. Figure 1 shows the experimental phase diagram.

The $c_{v}$ interval when the $\mathrm{H}$ phase is crossed in the I-H-N ${ }_{c}$ sequence (lines A and B in Fig. 1) is too small to allow the determination of the exponent $x$, obtained in the binary system. However, a characterization can be made by a direct comparison of the $L / c$ value obtained from Eq. (1), with known $c_{v}$ and $R_{e f f}=18.4 \AA$ $[9,13]$, and the $2 R_{t o t} / a$ value $\left(a=2 / \sqrt{ } 3 s^{-1}\right)$, as performed in refs. $[15,16]$. $R_{\text {tot }}$ is the "particle" radius, given by the sum of the extended dodecyl chain, $16.7 \AA$, and the polar head diameter, $4.6 \AA$. The two values $L / c$ and $2 R_{t o t} / a$ give the best definition of the "cylindrical particle" of the $\mathrm{H}$ phase in the directions $c$ and $a$, respectively.

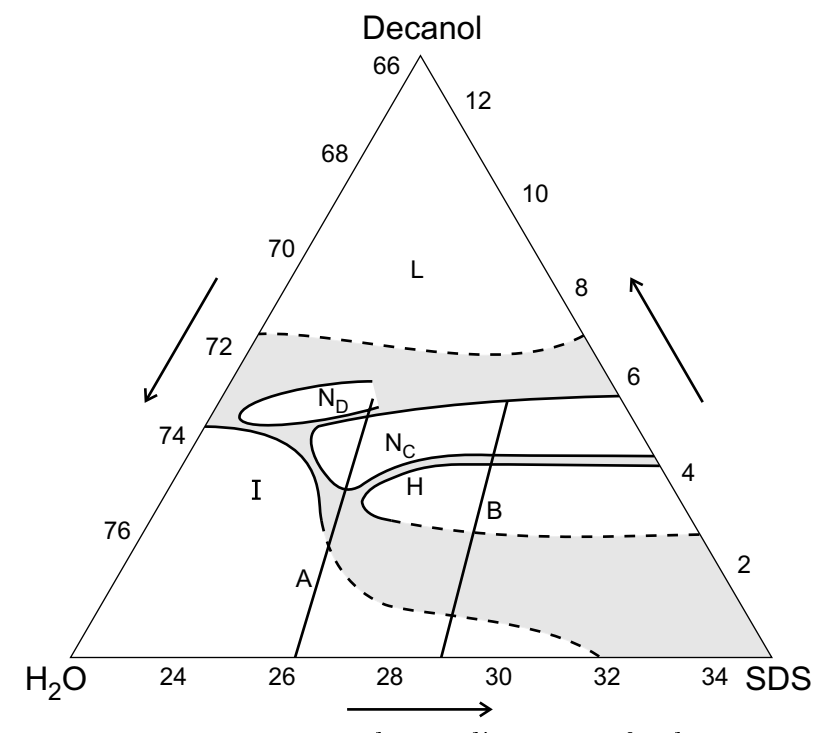

Figure 1. Ternary phase diagram of the system SLS/water/decanol as a function of weight composition from Quist et al [20], which includes the nematic domain previously localized by Amaral et al $[18,19]$ and the limits of the hexagonal phase given by Ekwall [2], when the $\mathrm{N}$ domain was not known. Results for lines A [15] and B [16] are presented in Fig. 2. The notation SDS is used for SLS in ref.[2] and [20].

Figure 2 (given also in [16]) gives $L / c$ and $2 R_{\text {tot }} / a$ values from three different data sets $[15,16,25]$, as a function of the micellar $c_{v}$, which includes both SLS and decanol. The three data sets correspond to different SLS:water molar ratios $\left(M_{w}\right)$, two in ternary systems, with $M_{w}=39.4$ (line A of Fig. 1 [15]) and 45.2 (line B of Fig. 1 [16]), and one in the binary system $\left(M_{w}=24.0[25]\right)$. The first two points for $M_{w}=39.4$ correspond to the $\mathrm{H}$ phase in coexistence with I phase.

It is evident from Fig. 2 that the condition $L / c=$ $2 R_{\text {tot }} / a$ is only attained in the $\mathrm{H}$ phase of the studied binary SLS/water system [25], in agreement with $a \sim c_{v}^{-1 / 3}$ behavior [9]. However $L / c$ values are close to 1 and much larger than $2 R_{\text {tot }} / a$ in the $\mathrm{H}$ phases of the ternary SLS/water/decanol system, for two different values of $M_{w}[15,16]$. It is seen that the addition of decanol deeply changes the characteristics of the $\mathrm{H}$ phase, which switches from particle finite/rigid to particle infinite/flexible behavior. The results for $M_{w}=39.4$ show even a tendency for increase in $L / c$ and small decrease in $2 R_{\text {tot }} / a$ with increase in decanol content.

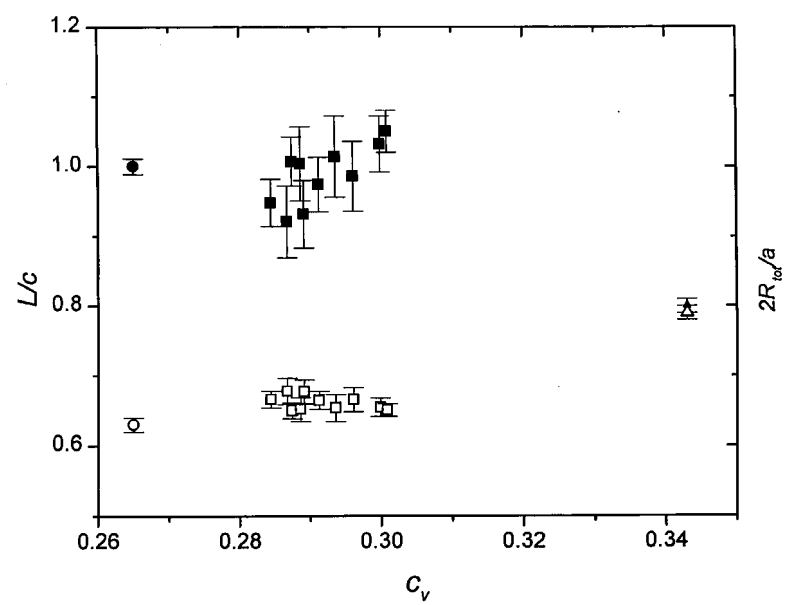

Figure 2. (Appearing also in [16]): $L / c$ (solid symbol) and $2 R_{\text {tot }} / a$ (open symbols) values in the $\mathrm{H}$ phases as a function of (SLS + decanol) volume concentration for three data sets, corresponding to three different values of the water: SLS molar ratio. Squares correspond to the results [15] with $M_{w}=39.4$, circles to a ternary system with $M_{w}=45.2[16]$ and triangles to a binary system with $M_{w}=24.0[25] . L / c$ values were obtained from Eq. (1), using $c_{v}$ and $R_{\text {eff }}=18.4 \AA$. $R_{\text {tot }}$ and $a$ are, respectively, the total particle radius (see text for details) and the hexagonal cell parameter.

It should be noted that the $R_{\text {eff }}$ value used to calculate $L / c$ was obtained in the binary system $[9,13]$. In order to further demonstrate the result in the ternary system, analysis of the electron density maps in the ternary system were also performed [16]. The maps of the ternary system [16] looked very similar to the maps [13] of the binary system regarding the micellar object. Only the fraction occupied by the micelle in the hexagonal cell changes, according to different $c_{v}$ and $a$ values of the phases. The ternary system has a hexagonal cell with considerably more water in the hexagonal plane.

A consistent interpretation of the cylindrical structure is obtained [13] by estimating $R_{\text {eff }}$ from the electron density maps using as a criterion the largest distance of the continuous contour from the micellar center. Results show [16] that, in fact, $R_{e f f}$ is slightly smaller in the ternary system. The average value is about $4 \%$ smaller than the value obtained in the binary system. The $R_{\text {par }}$ values however are within $1 \%$ the same as in the binary system. This indicates that the presence of decanol changes only slightly the micellar radius, and that the change in $R_{e f f}$ occurs in the polar shell, since decanol decreases its electron density. 
As far as the $L / c$ values are concerned, they are equal to 1 , within the evaluated uncertainties. It means that such ternary $\mathrm{H}$ phases are built up of infinite/flexible cylinders in hexagonal array.

In order to clarify the role of decanol, we analyze available theories of self-assembly and confront experimental and theoretical phase diagrams.

\section{Phase diagrams and theories of self-assembly}

The theories of self-assembly predict the sequence I $\left(\mathrm{N}_{c}\right)-\mathrm{H}$, as a function of increased particle volume fraction $v_{p}$, for cases of finite hard rods [21], persistent flexible rods [22] and wormlike micelles [23]. All these theories present phase diagrams as a function of $v_{p}$ with the same topology, seen in Fig. 3 . There is a triple point that separates I-H and I-N-H phase transitions. In the case of finite rigid objects, the phase diagram [21] is a function of the association free energy $\Phi$, which defines the particle length. In the case of flexible long rods, the phase diagram $[22,23]$ is a function of the ratio persistent length / micelle diameter $(P / D)$ with the axial ratio $(\mathrm{L} / \mathrm{D})$ as parameter $(\mathrm{D}=2 \mathrm{R})$. The direct I-H transition is expected in the limits of small rigid objects or very flexible long objects in I phase. In the former an increase in micellar length is expected at I - H transition [21].

The expected theoretical phase sequence $\mathrm{I}-\mathrm{N}_{c}-\mathrm{H}$ appears in the experimental phase diagram of the ternary system SLS/water/decanol, shown in Fig. 1, only for about $4 \mathrm{wt} \%$ of decanol and between 24.5 and $27 \mathrm{wt} \%$ of SLS. This means that only for those concentrations the micelles are in the correct range of length and rigidity to induce the appearance of the $\mathrm{N}_{c}$ phase with increase in SLS content.

Inverted phase sequence $\mathrm{I}-\mathrm{H}-\mathrm{N}_{c}$ occur, however, by increasing decanol content, upon SLS concentration larger than $26 \mathrm{wt} \%$. Such inversion must be due, therefore, to a specific effect of decanol, which possibly affects the growth and flexibility of the mixed micelles in a non-trivial way. The inversion occurs in the context of the complete sequence $\mathrm{I}-\mathrm{H}-\mathrm{N}_{c}-\mathrm{N}_{d}$ - L, which appears only with increase in decanol content. We shall not discuss now two small biaxial islands [29], not shown in Fig. 1 because they do not appear along the $\mathrm{A}$ and $\mathrm{B}$ lines, here analyzed in detail.

A better understanding of the decanol effect on micellar structure and phase transitions was recently obtained through the study of the phase sequence I - H $\mathrm{N}_{c}$ by investigating the ternary system varying $M_{d}$ for fixed values of the water: SLS molar ratio $\left(M_{w}\right)$. It was shown $[14,15]$ that the inverted sequence appears already for $M_{w}=45.2$ (line A of figure 1). For such high water content the $\mathrm{H}$ domain exists only for a very narrow interval, $M_{d} \sim 0.195$. So, the line A contains in fact the $\mathrm{H}$ phase in the sequence $\mathrm{I}-\mathrm{H}-\mathrm{N}_{c}$ and passes very near the triple point. The $\mathrm{H}$ phase's features over a larger range of $M_{d}$ values, by investigating samples at $M_{w}=39.4$ (line B of Fig. 2), was also performed [16] following the sequence $\mathrm{I}-\mathrm{H}-\mathrm{N}_{c}$.

The results discussed in the previous section 3 confirm a "crossover" from the finite/rigid model [21] to the infinite/flexible model $[22,23]$ for the $\mathrm{H}$ phase with an increase in the decanol content. This effect is probably due essentially to micellar growth with decanol addition. Such growth occurs in a slighter form (anisometries increasing from 2.4 to 3 ) in I phase [30] but must be greatly enhanced by coupling with orientational and positional order in the $\mathrm{H}$ phase [21-23].

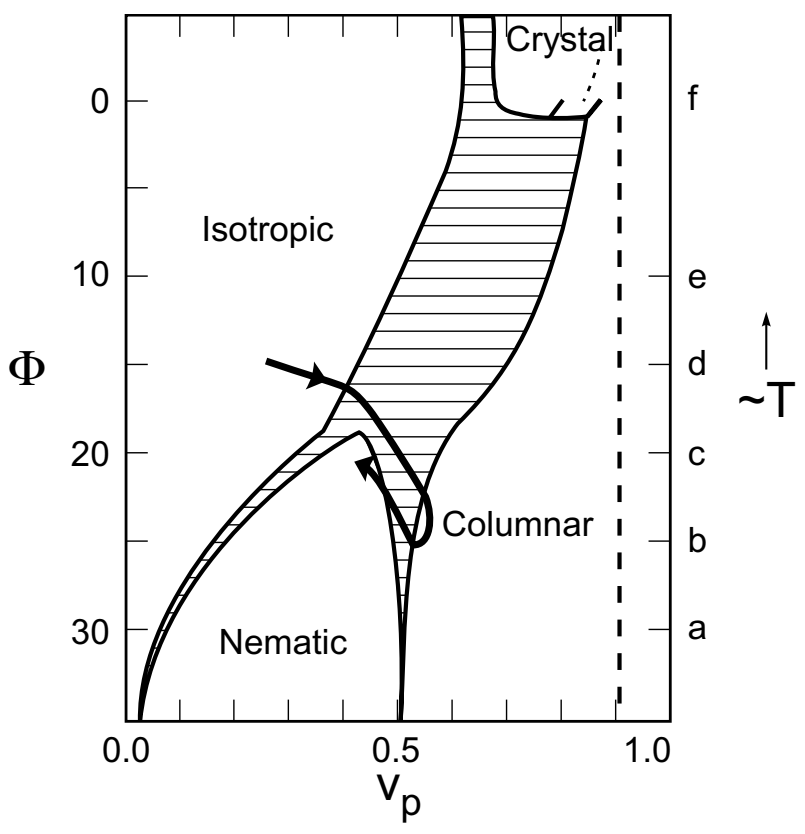

Figure 3. Theoretical phase diagrams for rods, taken from [21]. The complex path proposed in this work (and described in the text) corresponds to the curve drawn here around the triple point, allowing for the observed phase sequence I - $\mathrm{H}$ (columnar) - $\mathrm{N}_{c}$.

In the binary system micelles are short in I phase and longer in $\mathrm{H}$ phase, growing considerably in the $\mathrm{I}-\mathrm{H}$ coexistence range $[13,25]$. From X-ray results [13] the estimated length in phase $\mathrm{H}$ gives $\mathrm{L} / \mathrm{D}$ in the range 616. The behavior $a \sim c_{v}^{-1 / 3}$ assures [9] that micelles are in the finite rigid regime, and therefore must have a large persistence length, or $\mathrm{P} \sim \mathrm{L}$. No nematic phase exists in the binary system, so the system is above the triple point at the $\mathrm{I}-\mathrm{H}$ transition.

The theories of self-assembly that predict I - $(\mathrm{N})-\mathrm{H}$ phase transitions [21-23] are neither specific for ternary systems, nor deal with the specific role of decanol. However, the effect of decanol might be deduced through comparison of the experimental phase diagram of Fig. 1 with the theoretical predictions, shown in Fig. 3. The first point to emphasize is that decanol brings I - H transition to values of SLS concentration much smaller than 
without decanol. In the binary system I - H direct transition occurs [25] for $40 \mathrm{wt} \%$ of SLS, while it can occur for less than $26 \mathrm{wt} \%$ for about $3.5 \mathrm{wt} \%$ of decanol (see Fig. 1). This shows that the particle volume fraction $v_{p}$, which defines the transition point, is neither proportional to the SLS nor to the total amphiphile (SLS + decanol) weight concentration, and must greatly increase with decanol addition.

Let us first compare the experimental phase diagram with the phase diagram for rigid rod [21]. From the position of the triple point we conclude that the decanol content plays the role of the association free energy $\Phi$. A slight decrease in decanol (from $4 \mathrm{wt} \%$ to $3.7 \mathrm{wt} \%$ ) promotes a change from I-N-H to I-H behavior. It shows that increase in decanol should have the same effect of an increase in the absolute value of $\Phi$ (or alternatively a decrease in temperature). This is consistent with the effect of micellar growth with decanol addition mentioned before. From the theoretical phase diagram for rigid rods the $\mathrm{H}-\mathrm{N}$ line can only be crossed through a decrease in particle volume fraction $v_{p}$. The $\mathrm{H}-\mathrm{N}_{c}$ transition is experimentally obtained with increase in $c_{v}$ and $c_{v p a r}$ (since $M_{d}$ increases at constant $M_{w}$ ), but this does not mean necessarily an increase in $v_{p}$. With the constant $R_{\text {tot }}$ value used in Fig. 2, $v_{p}$ shows a slight increase with $M_{d}$. But if we allow for a small decrease in $R_{t o t}$, as indicated in the structural analysis that gave a decrease of $4 \%$ in $\mathrm{R}_{e f f}$, $v_{p}$ can actually decrease as $M_{d}$ increases. It is thus possible that, due to a combined effect of marked particle growth and small decrease in polar head size, $v_{p}$ decreases within the $\mathrm{H}$ domain, inducing the $\mathrm{H}-\mathrm{N}_{c}$ transition. The particle volume fraction in the hexagonal plane is clearly much smaller in the ternary than in the binary system, while the fraction in the fluid direction increases in the ternary system. So, decanol has the effect of initially increase $v_{p}$, due to micellar growth and increase in micellar length, but in excess produces a small decrease in $v_{p}$, due to its effect in decrease the micelle diameter.

To complete the picture it is necessary to take into acount that the nearby nematic phase $\mathrm{N}_{c}$ is made up of small micelles [31], while micelles are long in the $\mathrm{H}$ phase. This can be rationalized only admitting that micelles must become shorter at the $\mathrm{H}-\mathrm{N}_{c}$ transition and, conversely, that a large increase in micellar length must occur at the $\mathrm{N}_{c^{-}} \mathrm{H}$ transition. Such fact is qualitatively understandable, since micellar growth is triggered by both orientational and positional order [21-23]. What experimental results show is that, since anisometries are nearly equal in the I phase $(\mu \sim 3)$ before the $\mathrm{I}$ $\mathrm{H}$ transition [14,30] and in the $\mathrm{N}_{c}$ phase after the $\mathrm{H}$ - $\mathrm{N}_{c}$ transition [31], growth occurs much more due to positional ordering than due to orientational ordering. Enhancement of growth in the $\mathrm{H}$ phase, as compared to the $\mathrm{N}$ phase, arises theoretically [23] from details of polydispersity and intermicellar interactions.
The non-trivial I-H-N ${ }_{c}$ sequence in the SLS ternary system with increase in $M_{d}$ may be therefore understood on basis of a complex path through the theoretical phase diagram for rigid rods [21]. I - H transition occurs above the triple point, for small lengths and large volume fraction in I phase, with large increase in micellar length at $\mathrm{I}-\mathrm{H}$ coexistence. Along the $\mathrm{H}$ phase increase in $M_{d}$ corresponds to further micellar growth, arriving to the "infinite end-to-end" limit. This path goes, therefore, around the triple point (which corresponds to $\mu \sim 3$ for $v_{p} \sim 0.45$ ), with an increase in size and decrease in $v_{p} . \mathrm{H}-\mathrm{N}_{c}$ transition occurs below the triple point with a marked decrease in micellar length at the $\mathrm{H}-\mathrm{N}_{c}$ coexistence.

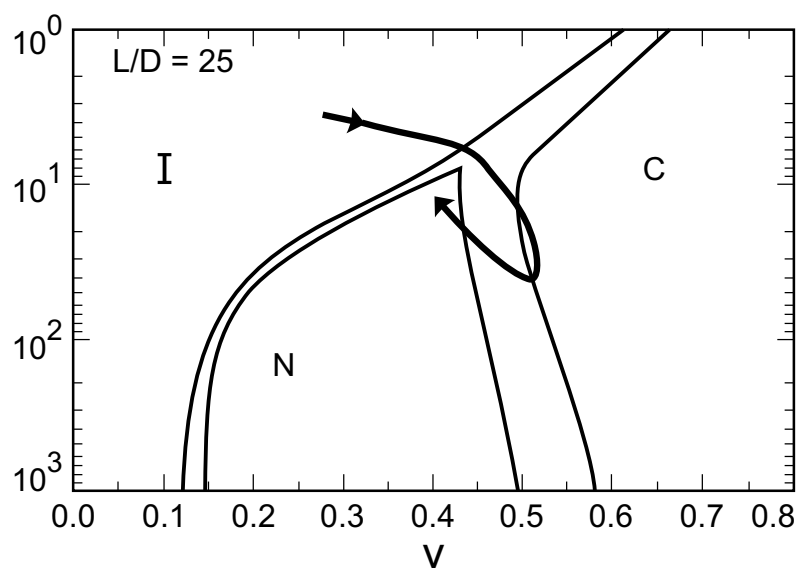

Figure 4. Theoretical phase diagram for flexible rods taken from [22]. Ordenate P/D (see text) in log scale as a function of volume fraction $\mathrm{V}$. The complex path proposed in this work (and described in the text) corresponds to the curve drawn here around the triple point, allowing for the observed phase sequence I - H (columnar) $-N_{c}$.

Let us now compare the experimental phase diagram also with the predictions for flexible rods $[22,23]$, since results showed that the $\mathrm{H}$ phase is in the "infinite/flexible" limit. It has been shown that the existence of the $\mathrm{N}$ phase requires a certain rigidity of semiflexible micelles. Comparison of the triple point of Fig. 1 with the theoretical phase diagram (Fig. 4) shows that increase in decanol content must induce an increase in the $\mathrm{P} / \mathrm{D}$ ratio of a long flexible rod. That means that increase in decanol should increase rod rigidity, and not promote flexibility. The micelles in the $\mathrm{H}$ phase of the binary system are quite rigid, since they have rigid behavior and are long $(\mathrm{L} / \mathrm{D} \sim 10)$. Therefore they have $\mathrm{P} \geq \mathrm{L}$. Increase in rigidity can be obtained either by increase in $\mathrm{P} / \mathrm{D}$ or decrease in L/D. Since decanol promotes miceller growth (increase in L/D), it must promote also marked increase in $\mathrm{P} / \mathrm{D}$ in order to allow the $\mathrm{H}-\mathrm{N}_{c}$ transition within the theoretical framework of flexible rods. Increase in rigidity of rod like micelles with decanol addition can be understood within the framework of the elastic bending energy of mixed micelles [24]. The surfactant parameter of de- 
canol $\left(p_{o} \sim 1\right)$, responsible for its bending energy at the micellar polar / apolar interface, is considerably larger than that of SLS $\left(p_{o} \sim 1 / 3\right)$, and the parameter of the mixed region will be also correspondingly larger [24]. Thus decanol promotes rigidity in rod like micelles, even if may eventually promote flexibility in planar membranes, due to the planar symmetry combined with decrease in charge of polar surfaces.

The direct $\mathrm{I}$ - $\mathrm{H}$ transition occurs above the triple point (which occurs for $\mathrm{P} / \mathrm{D} \sim 13$ for long flexible rods $[22,23])$. Increase in $M_{d}$ corresponds again to a path "turning around the triple point", with increase in L/D and $\mathrm{P} / \mathrm{D}$. The transition line $\mathrm{H}-\mathrm{N}_{c}$ is crossed also with a decrease in $v_{p}$, combined with increase in $\mathrm{P} / \mathrm{D}$. It should be remarked that, in one of the theoretical phase diagrams [22], it is possible a slightly re-entrant behavior (for $\mathrm{L} / \mathrm{D} \sim 25$ ), which allows to cross the $\mathrm{H}-\mathrm{N}$ line at fixed $v_{p}$, but with a very large increase in $\mathrm{P} / \mathrm{D}$ (of about 3 orders of magnitude). However a decrease in $v_{p}$ seems more plausible. Moreover, as the infinite limit is being approached, the occupancy in the hexagonal plane, given by $2 R / a$, which is clearly decreasing with $M_{d}$, plays the role of $v_{p}$.

In conclusion, the experimental facts can be explained within the framework of the theoretical phase diagrams for self-assembly systems, but with a rather complex path unifying both phase diagrams. The "infinite/flexible" limit behavior observed for the $\mathrm{H}$ phase of the ternary system cannot in fact be ascribed neither to "infinite" nor "flexible" micelles, since decanol promotes growth, but not flexibility. There must be therefore a limit on the validity of. Eq. (3a), possibly related to a minimum value of $2 \mathrm{R} / \mathrm{a}$ that is consistent with partition of water in the three dimensions. Bellow such limit, Eq. (3b) holds. Thus the "infinite/flexible" limit is reached in the ternary system because decanol is unable to form micelles on its own, being forced to enter on the SLS micelles, causing micellar growth and decrease in $2 R / a$. The real ternary system crosses the I - H line within the "finite/hard" framework, then micellar growth with increase in $M_{d}$ induces a passage to the "infinite" framework, which together with decrease in $v_{p}$ induces finally the crossing of the $\mathrm{H}-\mathrm{N}_{c}$ line, and this transition is again within the framework of the "finite/hard" limit.

\section{The $N_{c}-\left(N_{b}\right)-N_{d}$ phase se- quence}

This sequence has not been worked out yet theoretically within the framework of self-assembly theories, which consider the micelle symmetry as a priori defined, and exist up to now only for the $\mathrm{I}-\mathrm{N}_{c}-\mathrm{H}$ and I $-\mathrm{N}_{d}-\mathrm{L}$ sequences, that refer respectively to rods and discs. It is out of the scope of the present paper to review all other theoretical statistical approaches, which do not take self-assembly into account, and also do not account for the neighboring $\mathrm{H}$ and $\mathrm{L}$ phases with positional order, as well as the extensive work done by other groups in the second order phase transitions with temperature that occur in a particular system (potassium laurate/water/decanol).

However, the $\mathrm{N}_{c^{-}} \mathrm{N}_{d}$ transition has been shown experimentally $[19,24]$ to be a function of decanol:SLS molar ratio $\left(M_{d}\right)$ in systems with three different amphiphiles, occurring always around similar $M_{d}$ values. Furthermore, it was shown [32] that the system SLS/water/decanol has a first order phase $\mathrm{N}_{c}-\mathrm{N}_{d}$ transition at such $\mathrm{M}_{d}$ value. Two small biaxial islands have been later discovered [29], evidencing also first order transitions and even the existence of two different biaxial phases $\left(\mathrm{N}_{b+}\right.$ and $\left.\mathrm{N}_{b-}\right)$, strongly indicating micellar shape transformations. Clearly, $\mathrm{N}_{c}-\mathrm{N}_{d}$ direct phase transitions exist, and phase $\mathrm{N}_{b}$ does not occur necessarily in between.

We have developed an elastic bending theory for a "single micelle", based on the surfactant parameter $p_{o}$, which is able to account for shape transformations. It has been shown [28] that a form transformation from prolate spheroids to spherocylinders (SC) occurs, as the micellar anisotropy increases (for $\mu>1.8$ ), due to the bending energy of the polar/apolar interface. Such transformation is a pre-requisite for I - H transition, since the SC form allows polydisperse grow. It has also been shown [33] that it is energetically favored in SC mixed micelles that decanol stays in the body of the cylinder, leaving unmixed amphiphile in the hemispherical caps. The tendency of decanol to localize in the body of SC micelles occurs in order to decrease the electrostatic interaction between amphiphile polar heads, and is responsible for micellar growth with decanol addition. The shorter micelles in the $\mathrm{N}_{c}$ phase will have a larger decanol mole fraction in the body of the $\mathrm{SC}$ (here named $y$ ) than in the $\mathrm{H}$ phase, but still without decanol in the hemispherical caps. Only with further decanol increase, at the $\mathrm{N}_{c}-\mathrm{N}_{d}$ transition, the decanol of the cylindrical body imposes a marked change in micellar form (from SC to platelet), as extensively studied by Amaral et al [24]. A synthesis of the obtained results is shown in table 1 . The experimental $M_{d}$ value and the known $\mathrm{SC}$ anisometry $\mu$, at the $\mathrm{N}_{c}$ - $\mathrm{N}_{d}$ transition, give an "experimental" $y$, which can be compared to the predictions of the elastic bending model.

Table 1 shows a constant $y$ value for the three amphiphiles. Note that the $M_{w}$ values at the transition for the three amphiphiles are rather different [19], due to different micellar sizes and water binding. The elastic bending theory, for a single mixed micelle, correctly predicts [24] a transformation at this $y$ value for expected $p_{o}$ values of amphiphile and cosurfactant, without entering in the details of the intermediate forms between $\mathrm{SC}$ and discs, neither in the intermicellar interactions. 
This form transformation, besides coinciding with $\mathrm{N}_{c}$ $-\mathrm{N}_{d}$ transitions, occurs also in the I phase, as studied by detailed analysis [17] of curves of small angle X-ray scattering in the SLS/water/decanol system.

Table I. Experimental $M_{d}$ values (decanol/amphiphile molecular ratio), estimated anisometries $\mu$ of the SC and related $y$ values (decanol/amphiphile ratios in the body of the SC), at the $\mathrm{N}_{c}-\mathrm{N}_{d}$ transition, taken from [24]. SDS, KL and SLS are, respectively, the amphiphiles sodium decyl sulfate, potassium laurate and sodium dodecyl sulfate.

\begin{tabular}{|c|c|c|c|}
\hline & SDS & KL & SLS \\
\hline$M_{d}$ & $0.30 \pm 0.03$ & $0.38 \pm 0.02$ & $0.38 \pm 0.01$ \\
$\mu$ & $2.3 \pm 0.3$ & $3.0 \pm 0.3$ & $3.0 \pm 0.3$ \\
$\mathrm{y}$ & $0.65 \pm 0.03$ & $0.64 \pm 0.03$ & $0.64 \pm 0.03$ \\
\hline
\end{tabular}

This idea of shape transformation at the $\mathrm{N}_{c}-\left(\mathrm{N}_{b}\right)$ - $\mathrm{N}_{d}$ phase sequence inspired a proposal of considering the statistical mechanics of polydisperse uniaxial forms, which was worked out by Henriques \& Henriques [34], leading to a phase diagram with the typical topology of the I - N transitions, including the two uniaxial and one biaxial phases. Phase transitions with temperature are in this model correlated with different populations of the two uniaxial forms.

To sum up, the sequence $\mathrm{I}-\mathrm{H}-\mathrm{N}_{c}-\mathrm{N}_{d}-\mathrm{L}$ can be understood by a "superposition" of theories of selfassembly (which consider the micelle symmetry as a priori defined) with an elastic bending theory for a "single micelle" (which accounts for shape transformation). It remains to be worked out an extension of such elastic theory to a statistical ensemble of micelles.

\section{Acknowledgments}

The author thanks the contribution of the collaborators of the works here reviewed, particularly Drs. R. Itri, P. Mariani, G. Taddei, O. Santin Filho, V. Castelletto and C. V. Teixeira, and the financial support of PRONEX/CNPq/MCT and FAPESP.

\section{References}

[1] V. Luzzati, Biological Membranes, ed. D.Chapman, Academic Press, New York, pp. 1-123 (1968).

[2] P. Ekwall, Advances in Liquid Crystals, ed. G.H.Brown, Academic Press, London, vol.1, pp.1-142 (1975).

[3] J. N. Israelachvili, D.J. Mitchell, and B.W. Ninham, J. Chem. Soc. Faraday Trans.II 72, 1525 (1976).

[4] P. Mariani, V. Luzzati, and H. Delacroix, J. Mol. Biol. 204, 165 (1988).

[5] J.B. Forrest and L.W. Reeves, Chem. Rev. 81, 1 (1981).

[6] L.Q. Amaral, C.A. Pimentel, M.R. Tavares, and J.A. Vanin. J. Chem. Phys. 71, 2940 (1979).
[7] J. Charvolin, A.M. Levelut, and E.T. Samulski, J. Physique Lett. 40, L-587 (1979).

[8] L.J. Yu and A. Saupe, Phys. Rev. Lett. 45, 1000 (1980).

[9] L.Q. Amaral, A. Gulik, R. Itri, and P. Mariani. Phys. Rev. A 46, 3548 (1992).

[10] L.Q. Amaral, R. Itri, P. Mariani, and R. Micheletto, Liq. Crystals 12, 913 (1992).

[11] P. Mariani and L.Q. Amaral, Phys. Rev. E 50, 1678 (1994).

[12] P. Mariani, L.Q. Amaral, L. Saturni, and H. Delacroix, J. Phys. II (France) 4, 1393 (1994).

[13] R. Itri, L.Q. Amaral, and P. Mariani, Phys. Rev. E 54, 5211 (1996).

[14] R. Itri and L.Q. Amaral, Phys. Rev. E 58, 1173 (1998).

[15] C.V. Teixeira, R. Itri, and L.Q. Amaral, Langmuir 15, 936 (1999).

[16] O. Santin Filho, R. Itri, and L.Q. Amaral, J. Phys. Chem. 104, 959 (2000).

[17] C.V. Teixeira, R. Itri, and L.Q. Amaral, Langmuir 16, $6102(2000)$.

[18] L.Q. Amaral, M.E.M. Helene, D.R. Bittencourt, and R. Itri, J. Phys. Chem. 91, 5949 (1987).

[19] L.Q. Amaral and M.E.M. Helene, J. Phys. Chem. 92, 6094 (1988).

[20] P.O. Quist, B. Halle, and I. Furo, J. Chem. Phys. 95, 6945 (1991).

[21] M.P. Taylor and J. Herzfeld, Phys. Rev. A 43, 1892 (1991).

[22] R. Hentschke and J. Herzfeld, Phys. Rev. A 44, 1148 (1991).

[23] P. van der Schoot, J. Chem. Phys. 104, 1130 (1996).

[24] L.Q. Amaral, O. Santin Filho, G. Taddei, and N. VilaRomeu, Langmuir 13, 5016 (1997).

[25] R. Itri and L.Q. Amaral, J. Phys. Chem. 94, 2198 (1990).

[26] V. Castelletto, R. Itri, and L.Q. Amaral, J. Chem. Phys. 107, 638 (1997).

[27] V. Castelletto and L.Q. Amaral, J. Phys. Chem. B 103, 8877 (1999).

[28] G. Taddei and L.Q. Amaral, J. Phys. Chem. 96, 6102 (1992).

[29] P.O. Quist, Liq. Cryst. 18, 623 (1995).

[30] R. Itri and L.Q. Amaral, Phys. Rev. E 47, 2551 (1993).

[31] P.O. Quist, B. Halle, and I. Furo, J. Chem. Phys. 96, 3875 (1992).

[32] L.Q. Amaral, Liquid Crystals 7, 877 (1990).

[33] W.M. Gelbart, W.E. McMullen, A. Master, and A. Ben-Shaul, Langmuir 1, 101 (1985).

[34] E.F. Henriques and V.B. Henriques, J. Chem. Phys. 107, 8036 (1998). 